TRANSACTIONS OF THE

AMERICAN MATHEMATICAL SOCIETY

Volume 195,1974

\title{
REGULAR ELEMENTS IN RINGS WITH INVOLUTION
}

\author{
BY
}

\section{CHARLES LANSKI $(1)$}

ABSTRACT. The purpose of this paper is to determine when a symmetric element, regular with respect to other symmetrics, is regular in the ring. This result is true for simple rings, for prime rings with either Goldie chain condition, and for semiprime Goldie rings. Examples are given to show that these results are the best that can be hoped for.

The structure of a ring with involution satisfying the property that no two nonzero symmetric elements annihilate one another was determined for 2torsionfree rings by Lanski in [4], and in general, by Herstein in [2]. It happens, at least for semiprime rings which are 2-torsion-free, that this assumption on the symmetric elements implies that each nonzero symmetric element is regular in the whole ring. It is our purpose here to determine conditions sufficient to imply that any single symmetric element which annihilates no nonzero symmetric element is regular in the whole ring.

Throughout this work, $R$ will denote a ring with involution * and $S=$ $\left\{r \in R \mid r^{*}=r\right\}$, the set of symmetric elements of $R$. To avoid confusion, we may write $S(A)$ for the symmetric elements of some ring $A$, with involution. We shall say that an element $y$ is regular in $S$ to mean that $y t=0$ implies $t=0$, for $t \in S$.

We are interested in determining when an element $y \in S$, which is regular in $S$, must also be regular in $R$. Of course, we cannot hope to draw such a conclusion unless $R$ is semiprime. A counterexample can be found easily by taking $R \oplus N$, for $N$ a 2-torsion-free ring with trivial multiplication, and setting $x^{*}=$ $-x$ for $x \in N$. It is also easy to find examples where $R$ has 1 , and so $N$ is not a direct summand. Let $R=F[x, y, z] /\left(z^{2}, z x, z y\right)$, for $F$ a field with char $F \neq$ 2 , and set $z^{*}=-z, x^{*}=y$ and $y^{*}=x$.

One condition on $R$ which allows us to conclude that elements regular in $S$ are regular in $R$ is given in our first observation.

Received by the editors September 7, 1973.

AMS(MOS) subject classifications (1970). Primary 16A28; Secondary 16A12, 16A46, $16 A 40,16 A 38$.

(1) This work was supported by NSF Grant GP 38601.

Copyright $\bigcirc$ 1974, American Mathematical Society 
Proposition 1. Let $R$ be an algebraic algebra with 1 over a field $F$. If $y \in S$ is regular in $S$, then $y$ is regular in $R$.

Proof. Let $K=F \cap S$. Then either $K=F$ or $\operatorname{dim}_{K} F=2$, so $R$ is algebraic over $K$. If $p(x)$ is the minimal polynomial for $y$ over $K$, and if $p(0)=0$, then $0=p(y)=y f(y)$. But $f(y) \neq 0$, since $\operatorname{deg} f<\operatorname{deg} p$, and $f(y) \in S$ since the coefficients of $f$ are in $K \subset S$. This contradicts our assumption that $y$ is regular in $S$. Thus $p(0) \neq 0$, which implies that $y$ is invertible in $R$.

Let us note that if $y \in S$ is regular in $S$, but not in $R$, then $r(y)=\{x \in R \mid y x=$ $0\}$ is a right ideal of $R$ containing no symmetric elements. We focus our attention on prime rings containing a right ideal which intersects $S$ trivially. The case of char $R=2$ causes no difficulty.

Theorem 2. Let $R$ be a prime ring with char $R=2$. If $T$ is a nonzero right ideal of $R$, then $T \cap S \neq 0$.

Proof. If $T \cap S=0$, then for any $t \in T$ we must have $t S t^{*} \subset T \cap S=0$. Consequently, for $r \in R, t\left(r+r^{*}\right) t^{*}=0$. Since char $R=2$, we may write $t r t^{*}=$ $t r t^{*}$, which implies that $t R t{ }^{*} \subset T \cap S=0$. Since $R$ is prime, $t=0$. Therefore, if $T \neq 0$ we may conclude $T \cap S \neq 0$.

Corollary 3. Let $R$ be a prime ring with char $R=2$. Then each symmetric element which is regular in $S$, is regular in $R$.

Having eliminated the trivial case, we turn to prime rings whose characteristic is not 2. Suppose $R$ is such a ring and $T \neq 0$ is a right ideal of $R$ with $T \cap S=0$. Proceeding as in Theorem 2, we obtain, for $t \in T$, that $t S t^{*}=0$, and so $t\left(r+r^{*}\right) t^{*}=0$. Now we have $t r t^{*}=-t r^{*} t^{*}$, which implies that $\left(t r t^{*}\right)^{*}=-t r t^{*}$. Since $t R t^{*} \neq 0$ for $t \in T-0$, we may conclude that there is $t \in T-0$ with $t^{*}=$ $-t$. Once again, $t S t=0$ and $t r t=-t r^{*}$. For $r, w \in R$

$$
t r t w t=-\operatorname{trtw}^{*} t=t r{ }^{*} w^{*} t=-t\left(w t^{*} r\right) t=t w t r t
$$

and so $R$ is a prime ring satisfying a generalized polynomial identity. By results of Martindale [6], if $C$ is the extended centroid of $R$, then $W=R C$ is a primitive ring with minimal right ideal $e W$, such that $e W e$ is a finite dimensional division algebra over $C$. Also, the involution on $R$ extends to $W[7, \mathrm{pp} .511-$ 512]. Using results of Jacobson [3, Chapter 4], we may consider $W$ as a dense ring of linear transformations acting on a vector space $M$, over a division ring $D$ with involution, where $M$ has a nondegenerate bilinear form which is either Hermitian or skew-Hermitian. Furthermore, the involution on $W$ is the adjoint with respect to the bilinear form, and $W$ contains all continuous transformations of finite rank. Thus, when $R$ contains a right ideal intersecting $S$ trivially, we 
may assume the situation just described, and we shall do so when necessary, without further elaboration.

In our attempt to settle the question of regularity when $R$ is prime, we first give an affirmative answer when $R$ is simple.

Theorem 4. Let $R$ be a simple ring and assume that $y \in S$ is regular in $S$. Then $y$ is regular in $R$.

Proof. If $y$ is not regular in $R$, then $T=r(y) \neq 0$. Since $y(T \cap S)=0$, we may conclude that $T \cap S=0$. Should char $R=2$, we are done by Theorem 2 . So we may assume char $R \neq 2$, and we are in the situation described above. However, because $R$ is simple, $C=$ centroid of $R$, so $W=R C=R$. Suppose that $R$ is a finite dimensional algebra over $C$. Then $R=M_{n}(D)$, and $y$ is either regular in $R$, or not regular in $S$, by Proposition 1. Thus, $R$ is infinite dimensional over $C$, and so, we may assume that $M$ is infinite dimensional over $D$. Since $R$ is simple, it is precisely the collection of all continuous transformations of finite rank. We now show that in this situation no element of $S$ can be regular in $S$.

Let $y \in S-(0)$ and write $y=\sum_{i=1}^{n}\left(, t_{i}\right) x_{i}$ where $($,$) is the bilinear form$ on $M \times M$, and $t_{i}$ and $x_{i}$ are in $M[3$, p. 74]. An easy computation shows that $((, t) x)^{*}= \pm(, x) t$, depending on whether $($,$) is Hermitian or skew-Hermitian.$ Also, for $m \in M-0$, codim $m^{\prime}=1$, where $m^{\prime}=\{w \in M \mid(m, w)=0\}$. This follows since there is some $n \in M$ with $(n, m) \neq 0$, and so, for $v \in M_{0}\left(v-(v, m)(n, m)^{-1} n, m\right)=0$. One can therefore choose $t, x \in\left(\bigcap_{i=1}^{n} x_{i}^{\prime}\right)$, with $x$ and $t$ independent over $D$, since $\operatorname{dim}_{D} M$ is infinite. Set $s=(, x) t \pm(, t) x$, picking the appropriate sign to make $s$ symmetric. Clearly $s \neq 0$, but $y s=0$, for

$$
\begin{aligned}
y s & =\left(\sum_{i=1}^{n}\left(, y_{i}\right) x_{i}\right) \circ((, x) t \pm(, t) x) \\
& =\left(\sum_{i=1}^{n}\left(, y_{i}\right) x_{i}, x\right) t \pm\left(\sum_{i=1}^{n}\left(, y_{i}\right) x_{i}, t\right) x=0
\end{aligned}
$$

by the choice of $x$ and $t$. Consequently, since $y$ is given to be regular in $S$, this situation cannot occur, so $y$ must be regular in $R$, proving the theorem.

We now present an example which shows that Theorem 4 cannot be extended to prime, in fact primitive, rings. The essential idea for the definition of the ring we obtain is due to $D$. Estes.

Let $F$ be a field with char $F \neq 2$, and $V$ a vector space over $F$ with basis $y_{0}, y_{1}, y_{2}, \cdots, y_{k}, \cdots$. Define a bilinear form $():, V \times V \rightarrow F$ on basis elements as follows: $\left(y_{2 i}, y_{2 i+1}\right)=1,\left(y_{2 i+1}, y_{2 i}\right)=-1$, and all other "products" of basis elements are zero. Extending bilinearly to $V \times V$, it is clear that $($, is skew-symmetric and nondegenerate. 
Let $H \subset \operatorname{Hom}_{F}(V, V)$ be the subring generated by $\{(, v) u \mid v, u \in V\}_{0} H$ is precisely the subring of all continuous transformations of finite rank [3, p. 75], and is a primitive ring. Note that for $(, v) v \in H$ and $t, w \in V$ we have

$$
\begin{aligned}
(t(, u) v, w) & =((t, u) v, w)=(t, u)(v, w) \\
& =-(t, u)(w, v)=(t,-(w, v) u) \\
& =(t, w(-(, v) u)) .
\end{aligned}
$$

This computation shows that $((, u) v)^{*}=-(, v) u$, where ${ }^{*}$ denotes the adjoint of $(, u) v$. Clearly, ${ }^{*}$ is an involution on $H$.

Define $T \in \operatorname{Hom}_{F}(V, V)$ by setting

$$
y_{2 i} T=y_{2 i+2}, \quad y_{1} T=0, \quad y_{2 i+1} T=y_{2 i-1},
$$

and extending to $V$ linearly. We claim that $T^{*}=T$. To see this, it is enough to show that $\left(y_{2 i} T, y_{2 j+1}\right)=\left(y_{2 i}, y_{2 j+1} T\right)$, since $($,$) is skew-symmetric, T$ preserves subscripts $\bmod 2$, and $\left(y_{i}, y_{j}\right)=0$ for $i \equiv j(\bmod 2)$. Clearly, $\left(y_{2 i} T, y_{1}\right)$ $=\left(y_{2 i+2}, y_{1}\right)=0=\left(y_{2 i}, 0\right)=\left(y_{2 i}, y_{1} T\right)$. Thus we may assume $j \geq 1$ in computing

$$
\left(y_{2 i} T, y_{2 j+1}\right)=\left(y_{2 i+2}, y_{2 j+1}\right)= \begin{cases}1 & \text { if } j=i+1 \\ 0 & \text { if } j \neq i+1\end{cases}
$$

and

$$
\left(y_{2 i}, y_{2 j+1} T\right)=\left(y_{2 i}, y_{2 j-1}\right)= \begin{cases}1 & \text { if } j=i+1 \\ 0 & \text { if } j \neq i+1\end{cases}
$$

Consequently, $T^{*}=T$ as claimed.

Note also, that for $(, u) v \in H,((, u) v) \circ T=(, u) v T \in H$, and for $w \in V$, $(w)(T \circ(, u) v)=(w T, u) v=(w, u T) v=w((, u T) v)$. Thus, $T \circ(, u) v \in H$, so $H$ is an ideal of the subring $R$ of $\operatorname{Hom}_{F}(V, V)$ generated by $H$ and $T, R$ is primitive since $V$ is irreducible as an $H$ module. Also, the adjoint * is an involution on $R$. This latter fact is easily verified using the computations above.

We claim that $T$ is not regular in $R$, but is regular in $S$. For the first part, observe that $\left((, v) y_{1}\right) \circ T=0$ for all $v \in V$, since $y_{1} \in \operatorname{Ker} T$. To show that $T$ is regular in $S$ we need to know that $f(T) \notin H$ for any nonzero polynomial $f(x) \in F(x)$. This is equivalent to showing that $f(T)=f_{0}+f_{1} T+\cdots+f_{n} T^{n}$ has infinite rank. Now the image of $f(T)$ contains $\left(y_{0}\right) f(T)=f_{0} y_{0}+f_{1} y_{2}+\cdots$ $+f_{n} y_{2 n}, y_{2 n+2} f(T)=f_{0} y_{2 n+2},+\cdots+f_{n} y_{4 n+2}$, and in general, the independent elements $\left\{\left(y_{(2 n+2) k}\right) f(T) \mid k \geq 0\right\}$. Consequently, if $r \in R$ and $r T=0$, then writing $r=b+$ $p(T), 0=b T+p(T) T$. As we have seen, $b T \in H$, thus $p(T) T \in H$, has finite rank, so $p(T)=0$, and $r \in H$. But the kernel of $T$ is spanned by $y_{1}$, so if $b T=0$, 
we must have $b=(, v) y_{1}$ for $v \in V$. Such an $b$ cannot be symmetric, since $\left((, v) y_{1}\right)^{*}=-\left(, y_{1}\right) v$, and $(, v) y_{1}=-\left(, y_{1}\right) v$ implies $v=f y_{1}$, for some $f \in F$. Thus $f\left(, y_{1}\right) y_{1}=-f\left(, y_{1}\right) y_{1}$, or $2 f=0$. Since char $f \neq 2, f=0$. In conclusion, no symmetric element of $R$ annihilates $T$, so $T$ is regular in $S$.

Although this example shows that Theorem 4 is not true for prime rings, we can obtain the result of the theorem by placing suitable chain conditions on these rings. We shall show that a prime ring satisfying either of the Goldie chain conditions, or a semiprime Goldie ring, satisfies the conclusion of Theorem 4. As a preliminary special case we consider prime rings satisfying a polynomial identity. It is well known [1] that such rings are Goldie rings.

Theorem 5. If $R$ is a prime ring satisfying a polynomial identity, then any $y \in S$ which is regular in $S$ is regular in $R$.

Proof. With the given hypothesis, it is known [8] that the localization of $R$ at its nonzero central elements, say $\bar{R}$, is a simple algebra, finite dimensional over its center $F$, the field of fraction of the center, $Z$, of $R$. Also, the involution on $R$ extends naturally to $\bar{R}$, and $\bar{R}$ is also finite dimensional as an algebra over $K=F \cap S(\bar{R})$. Assume that $y \in S(R)$ is not regular in $R$, so $y$ is not regular in $\bar{R}$. Using the minimal polynomial for $y$ over $K$, we may write $0=$ $c_{1} y+c_{2} y^{2}+\cdots+c_{n} y^{n}$, for $c_{i} \in K$ and $c_{n} \neq 0$. Clearly, each $c_{i}=a_{i} b^{-1}$, for $a_{i}, b \in Z$. From the fact that $a_{i} b^{-1} \in S(\bar{R})$, it follows that $a_{i} b^{*}=b a_{i}^{*} \in S(R) \cap Z$. Rewritting the above expression gives

$$
0=b^{*} b\left(b^{-1} a_{1} y+\cdots+b^{-1} a_{n} y^{n}\right)=\left(b^{*} a_{1}\right) y+\cdots+\left(b^{*} a_{n}\right) y^{n} .
$$

Consequently, $0=\left(b^{*} a_{1}+\cdots+b^{*} a_{n} y^{n-1}\right) y$ where the left factor is in $S$ and is not zero by the minimality of the first relation. Thus, if $y$ is not regular in $\bar{R}$, it cannot be regular in $S(R)$, which establishes the theorem.

The condition imposed on $R$ in Theorem 5 is a little stronger than necessary, but in view of our earlier example some additional hypothesis on $R$, besides being prime, is required. Essentially, what is required is that the central closure of $R$ has no infinite collection of orthogonal idempotents. Conditions on $R$ sufficient to ensure this situation are given in our next theorem.

Theorem 6. Let $R$ be a prime ring such that either $R$ bas no infinite direct sums of right ideals, or $R$ bas the ascending chain condition on right annibilators. If $y \in S$ is regular in $S$, then $y$ is regular in $R$.

Proof. Should char $R=2$, then we are done by Corollary 3. Assume that char $R \neq 2$ and that $y \in S$ with $r(y) \neq 0$. If $r(y) \cap S=0$ we may apply the remarks preceding Theorem 4 and consider $W=R C$, the central closure of $R$. If 
$W$ is finite dimensional over $C$, and so over $e W e$, then $R$ is an order in $M_{n}(e W e)$. Since $e W e$ is finite dimensional over $C, W$ satisfies a polynomial identity with integer coefficients. $R$ satisfies the same identity by restriction, so we may apply Theorem 5 to conclude that $y$ is not regular in $S$. Therefore, we may assume that $W$ is not finite dimensional over $C$, and so representing $W$ as a ring of continuous transformations on $M$ over $D$, we have that $M$ is infinite dimensional over $D$. We will show that this assumption is incompatible with either chain condition on $R$.

Denote the bilinear form on $M$ by $($,$) , and recall that W$ contains all continuous transformations of finite rank. It is necessary to know that $W$ contains an infinite collection of orthogonal idempotents. This result is known and can be obtained in several ways. We indicate one method of construction in the following. Choose $x_{1} \in M-0$, and $y_{1}$ such that $\left(x_{1}, y_{1}\right)=1$. Then $e_{1}=\left(, y_{1}\right) x_{1}$ is an idempotent, since

$$
e_{1}^{2}=\left(\left(, y_{1}\right) x_{1}, y_{1}\right) x_{1}=\left(, y_{1}\right)\left(x_{1}, y_{1}\right) x_{1}=\left(, y_{1}\right) x_{1}=e_{1}
$$

and $e_{1} \in W$ since it is continuous of rank 1. As pointed out in the proof of Theorem 4 , codim $x^{\prime}=1$ for any $x \in M-0$, so we may choose $y_{2} \in x_{1}^{\prime}-0$, and find $x_{2}$, with $\left(x_{2}, y_{2}\right)=1$. Then $e_{2}=\left(, y_{2}\right) x_{2}$ is an idempotent in $W$ and $e_{1} e_{2}=0$, by the choice of $y_{2}$. Given $e_{1}, \cdots, e_{n}$ with $e_{i}^{2}=e_{i}=\left(, y_{i}\right) x_{i}$, and $e_{i} e_{j}=0$ for $i<j$, pick $y_{n+1} \in x_{1}^{\prime} \cap \ldots \cap x_{n}^{\prime}, y_{n+1} \neq 0$, and find $x_{n+1}$ with $\left(y_{n+1}, x_{n+1}\right)=1$. Then $e_{n+1}=\left(, y_{n+1}\right) x_{n+1}$ is an idempotent in $W$ with $e_{i} e_{n+1}=0$ for $i \leq n$. Continuing in this manner, we obtain an infinite collection of idempotents $\left\{e_{i}\right\}$ with $e_{i} e_{j}=0$ for $i<j$. This condition on the $\left\{e_{i}\right\}$ will suffice, but it is easy to replace the $\left\{e_{i}\right\}$ with an orthogonal collection $\left\{f_{i}\right\}$, defined by $f_{1}=e_{1}, f_{2}=e_{2}\left(1-f_{1}\right)$, and in general, $f_{n}=e_{n}\left(1-f_{1}-f_{2}-\cdots-f_{n-1}\right)$.

Before proceeding, it is important to observe that any nonzero left or right ideal of $W=R C$, intersects $R$ nontrivally. Recall that for any finite subset $\left\{c_{1}, \cdots, c_{n}\right\} \subset C$, there is some nonzero ideal $U$ of $R$ with $c_{i} U \subset R$ and $U c_{i} \subset$ $R$ for each $i$ [6]. If $w \in W, w \neq 0$ and $w=r_{1} c_{1}+\cdots+r_{n} c_{n}$, choose an ideal $U$ as just described for the $c_{i}$. Then

$$
w U=\sum\left(r_{i} c_{i}\right) U=\sum r_{i}\left(c_{i} U\right) \subset R
$$

and

$$
U w=U \sum r_{i} c_{i}=\sum U\left(r_{i} c_{i}\right)=\sum\left(U r_{i}\right) c_{i}\left(U r_{i}\right) \subset \sum c_{i} U \subset R_{\bullet}
$$

Lastly, recall that since $R$ has an involution, either chain condition in the hypothesis holds for left ideals as well as right ideals. 
For $\left\{f_{i}\right\}$, an infinite set of orthogonal idempotents, $\Sigma W f_{i}$ is an infinite direct sum of left ideals in $W$. If $W f_{i} \cap R=L_{i}$, then $\Sigma L_{i}$ is an infinite direct sum of left ideals of $R$. Consequently, if $R$ satisfies the direct sum condition, we are forced to conclude that $W$ is finite dimensional over $D$, and the theorem is proved under this condition.

Finally, assume that $R$ satisfies the ascending chain condition on right annihilators. Clearly $r_{R}\left(\sum_{i=1}^{\infty} L_{i}\right) \subset r_{R}\left(\sum_{i=2}^{\infty} L_{i}\right) \subset \cdots$, for the $L_{i}=W f_{i} \cap R$ as above. To contradict $W$ infinite dimensional over $D$, we need only show that these inclusions are proper. This will be done for the first inclusion, the others being similar. From the orthogonality of $\left\{f_{i}\right\}$, it is clear that $\left(\Sigma_{i=2}^{\infty} W f_{i}\right) f_{1} W=0$. Hence $\left(\Sigma_{i=2}^{\infty} L_{i}\right)\left(f_{1} W \cap R\right)=0$, and $T=f_{1} W \cap R \neq 0$. Thus $T \subset r_{R}\left(\Sigma_{i=2}^{\infty} L_{i}\right)$, and it suffices to show that $L_{1} T \neq 0$. Let $U$ be a nonzero ideal of $R$ with $f_{1} U C$ $R$ and $U f_{1} \subset R$. Then $U f_{1} \subset L_{1}$ and $f_{1} U \subset T$, so if $L_{1} T=0$ then we must have $\left(U f_{1}\right)\left(f_{1} U\right)=0$. But then $U f_{1}^{2} U=U f_{1} U=0$. Since $W$ is a prime ring, $f_{1} U=0$, contradicting $f_{1} \neq 0$. Thus $L_{1} T \neq 0$, the above inclusion is proper, and the theorem is established.

One may ask whether Theorem 6 holds for semiprime rings. Our next example shows that the chain condition on annihilators is not sufficient to obtain the conclusion of Theorem 6 for semiprime rings.

For any field $F$, let $A$ be the free algebra $F\{x, y\}$ modulo the ideal generated by $x^{2}$. It is known that $A$ is a prime ring and if $c d=0$ for $c, d \in A$, then $c \in A x$ and $d \in x A$. Thus $r(c) \neq 0$ implies that $r(c)=x A$ for $c \neq 0$. Consequently, $A$ satisfies the ascending chain condition on left and on right annihilators. Let $R=A \oplus A^{0}$, where $A^{0}$ is the opposite ring of $A$. Define an involution by $(a, b)^{*}$ $=(b, a)$. Then $R$ is a semiprime ring with involution, and it is clear that $R$ satisfies the ascending chain condition on right annihilators. Also $S(R)=\{(a, a) \mid a \in$ $A\}$. Consider $(y x, y x) \in S$. Should $0=(y x, y x)(a, a)=(y x a$, ayx), we would have $y x a=0$ and $a y x=0$. But the second relation is possible in $A$ only if $a=0$. Thus $(y x, y x)$ is regular in $S$, but not in $R$, since $(y x, y x)(x, 0)=0$. Note that in $R,\left\{T_{i}=\left(y^{i} x A, A x y^{i}\right)\right\}$ is an infinite collection of right ideals whose sum is direct.

This example brings us to our last result.

Theorem 7. Let $R$ be a semiprime Goldie ring. If $y \in S$ is regular in $S$, then $y$ is regular in $R$.

Proof. If $R$ is prime, we are done by applying Theorem 6. Otherwise, there exists a finite set $\left\{P_{1}, P_{2}, \cdots, P_{n}\right\}$ of nonzero maximal annihilator ideals whose intersection is zero. These ideals are prime, and each factor ring $R / P_{i}=R_{i}$ is a prime Goldie ring. (See [1] for details.) Since $R$ can be represented as a sub- 
ring of $R_{1} \oplus \ldots \oplus R_{n}$, it suffices to prove that $y+P_{i}$ is regular in $R_{i}$ for $y$ a regular element of $S$.

If $P_{i}=\operatorname{Ann} A_{i}$, we may assume that $A_{i}=\operatorname{Ann} P_{i}$, and so, each $A_{i}$ is a minimal annihilator ideal. We consider two cases, depending on whether $P_{i}^{*}=$ $P_{i}$ or $P_{i}^{*} \neq P_{i}$

First assume that $P_{i}^{*} \neq P_{i}$, and for convenience, set $P_{i}=P$ and $A_{i}=A$. Since $A \cap P=0, A+P$ is a nonzero ideal in the prime Goldie ring $R / P$, so contains a regular element $c+P$ of $R / P$, where $c \in A$. Suppose $y$ is a regular element of $S$, but $(y+P)(r+P)=P$ for some $r \notin P$. Then $\left(r^{\prime}+P\right)(y+P)=P$ for some $r^{\prime} \notin P$, since $R / P$ is a prime Goldie ring. It follows that yrc $\in P \cap A=$ 0 and $c r^{\prime} y \in P \cap A=0$. Thus $r(y) \cap A \neq 0$ and $l(y) \cap A \neq 0$. As a minimal annihilator ideal, $A$ is prime as a ring $[1$, p. 83], and so,

$$
x(y) \cap l(y) \cap A=(f(y) \cap A) \cap(l(y) \cap A) \neq 0 .
$$

Let $B=l(y) \cap r(y) . B^{*}=B$ follows from $l(y)^{*}=r(y)$, so for $b \in B, b+b^{*} \epsilon$ $B$. This implies that $y\left(b+b^{*}\right)=0$, forcing $b^{*}=-b$. Consequently, $(B \cap A)^{*} C$ $B \cap A^{*} \cap A$. But if $A$ is a minimal annihilator ideal, so is $A^{*}$, with $A^{*}=$ $\operatorname{Ann}\left(P^{*}\right)$ and $P^{*}=\operatorname{Ann}\left(A^{*}\right)$, by choice of $A$ and $P$. By definition, $A^{*} P^{*}=0$, so $A^{*} C^{-} P$ or $P^{*} \subset P$. Our assumption that $P^{*} \neq P$ forces $A^{*} \subset P$. Thus $A \cap A^{*} C$ $A \cap P=0$, from which we obtain $A \cap B \subset\left(B \cap A \cap A^{*}\right)^{*}=B \cap A \cap A^{*}=0$, contradicting $B \cap A \neq 0$. Therefore, we are forced to conclude that $y+P$ is regular in $R / P$.

For the second case we assume $P^{*}=P$. Now $R / P$ has the induced involution $(r+P)^{*}=r^{*}+P$. Suppose that $t+P$ is symmetric in $R / P$ and $y t \in P$, for $y \in S$ and regular in $S$. Then $y t A=0$, and it follows that $y t(A \cap S) t^{*}=0$, and so, $t(A \cap S) t^{*}=0$. As in the first case, since $R / P$ is a prime Goldie ring, we can find $c \in A$ with $c+P$ regular in $R / P$. Of course, $w=c c^{*} \in A \cap S$ and $w+$ $P$ is regular in $R / P$. Since $w(A \cap S) w \subset A \cap S$, we may write $0=$ $w t w(A \cap S) w t^{*} w$. Consider this relation $\bmod P$, and let $d=w t w+P$. Note that $d^{*}=(w t w+P)^{*}=w t^{*} w+P=w t w+P=d$, since by assumption, $t-t^{*} \in P$. Thus in $R / P, d(A \cap S+P) d=0$. We claim that $d S((A+P) / P) d=0$. This follows easily, since if $a+P=a^{*}+P$ for $a \in A$, then $A^{*}=A n n P^{*}=A n n P=A$ forces $a-a^{*} \in P \cap A=0$, and $a \in S(R)$. Note that we actually have $(A \cap S)+P=$ $S(A+P / P)$. But $(A+P) / P$ is a semiprime subring of $R / P$ and $d \in S((A+P) / P)$, so $d S((A+P) / P) d=0$ forces $d=0[5, \mathrm{p} .537]$. That is. $w t w \in P$. The regularity of $w+P$ in $R / P$ implies that $t \in P$.

In summary, when $P^{*}=P$ and $y \in S$ is regular in $S$, then $y+P$ annihilates no symmetric element of $R / P$. In other words, $y+P \in S(R / P)$ is regular in $S(R / P)$. Since $R / P$ is a prime Goldie ring, $y+P$ must be regular in $R / P$ by Theorem 6 , and the proof of the theorem is complete. 


\section{REFERENCES}

1. I. N. Herstein, Topics in ring theory, Univ. of Chicago Press, Chicago, Ill., 1969. MR $42 \# 6018$.

2. - On rings with involution, Canad, J. Math. (to appear).

3. N. Jacobson, Structure of rings, 2nd ed., Amer. Math. Soc. Colloq. Publ., vol. 37, Amer. Math. Soc., Providence, R. I., 1964. MR 36 \#5158.

4. C. Lanski, Rings with involution whose symmetric elements are regular, Proc. Amer. Math. Soc. 33 (1972), 264-270. MR 45 \#1971.

5. - On the relationship of a ring and the subring generated by its symmetric elements, Pacific J. Math. 44 (1973), $581-592$.

6. W. S. Martindale III, Prime rings satisfying a generalized polynomial identity, J. Algebra 12 (1969), 576-584. MR 39 \#257.

7. - Prime rings with involution and generalized polynomial identities, $\mathrm{J}$. Algebra 22 (1972), 502 - 516. MR 46 \#5371.

8. L. Rowen, Some results on the center of a ring with polynomial identity, Bull. Amer. Math. Soc. 79 (1973), $219-223$.

DEPARTMENT OF MATHEMATICS, UNIVERSITY OF SOUTHERN CALIFORNIA, LOS ANGELES, CALIFORNIA 90007 